Short Report

\title{
Yoga: A Holistic Approach to Addiction Treatment and Recovery
}

\section{Dorothy S Greene *}

Associate professor in the department to Social Work, East Tennessee State University, Johnson City; E-Mail: greeneds@etsu.edu

* Correspondence: Dorothy Greene; E-Mail: greeneds@etsu.edu

Academic Editor: Viann N. Nguyen-Feng

Special Issue: Yoga and Mindfulness

OBM Integrative and Complementary Medicine 2021, volume 6, issue 4

doi:10.21926/obm.icm.2104047
Received: July 17, 2021

Accepted: November 09, 2021

Published: November 16, 2021

\begin{abstract}
Yoga is increasingly becoming a strategy for wellness in the United States. A 2016 survey found that nearly 37 million Americans practice yoga and an additional 12 million say they intend to try it in the coming year. Yoga is also being studied as an intervention for a number of physical and behavioral health disorders. This short report examines yoga's utility as a treatment or adjunctive tool for addiction recovery. Because addiction is characterized as a biopsychosocial-spiritual disorder and yoga is a holistic practice focusing on the integration of body, mind, and spirit, It is logical to test yoga's efficacy on the treatment and recovery of addiction. Yoga's positive effect on craving and negative mood states make it a promising modality for addiction recovery.
\end{abstract}

\section{Keywords}

Yoga; addiction; mindfulness; recovery; substance use disorder 


\section{Introduction}

Addiction is a complex disorder often characterized as a biological, psychological, social, environmental, and spiritual disorder affecting all aspects of an individual's life. For example, the American Society of Addiction Medicine [1] defines addiction as "a treatable, chronic medical disease involving complex interactions among brain circuits, genetics, the environment, and an individual's life experiences. People with addiction use substances or engage in behaviors that become compulsive and often continue despite harmful consequences" (para 1).

Many behavioral health disciplines and mutual aid recovery groups consider addiction from a biopsychosocial-spiritual perspective [2]. The most common mutual aid addiction recovery groups are based on the 12-steps of Alcoholics Anonymous (AA) and Narcotics Anonymous (NA), and 12step oriented groups conceptualize addiction as a physical, mental, and spiritual disease. For instance, NA [3] describes the physical aspect of addiction as the compulsive use of substances and the inability to stop or control use. The mental aspect of addiction is characterized by NA as the obsession to use, or craving, even when the person's life has been significantly harmed. Craving is one of the 11 diagnostic criteria for substance use disorder (SUD) [4], and craving may be associated with relapse risk. For example, Schneekloth et al. [5] found that an increased score on the Penn Alcohol Craving measure was significantly associated with increased relapse rates. Finally, according to NA, the spiritual deficit present in addiction is self-centeredness. Alcoholics Anonymous contends that sobriety will be precarious until the spiritual malady is overcome [6].

A commonly articulated truism in the mutual aid recovery community is "you gotta give up old people, places, and things" to remain sober. One of the most challenging aspects of early recovery is letting go of one's social network. When and individual's social network is rooted in recreational substance use, sustained recovery is unlikely. Like mutual aid recovery communities, the yoga community can serve as a healthy and supportive alternative social network and healing alternative. Given the commonly held perspective of addiction as a biopsychosocial-spiritual disorder, it seems logical to apply a holistic approach to healing addiction.

Yoga is gaining popularity as an intervention in addiction treatment and recovery, and science is beginning to support its efficacy, at least as an adjunctive tool for addiction recovery [7]. While the literature is still growing relative to yoga as an intervention for addiction treatment and recovery, and much of the available research has methodological flaws, most scientists agree: Yoga is a promising intervention as an alternative and/or complimentary practice for those with SUDs and may prove to have significant clinical utility as the research grows [8-12].

Yoga is so popular in the United States that a 2016 national poll found that 36.7 million Americans practice yoga, and 34 percent of Americans say they intend to try yoga in the next 12 months [13]. This article examines yoga and its usefulness for supporting individuals recovering from SUDs and addiction.

\section{What is Yoga?}

The term yoga has its roots in an ancient Indian language called Sanskrit. Yuj (yoga) literally means to join, unite, or yolk. This union of body, mind, and breath (or life force) is achieved through the eight limbs of yoga. For the purpose of this article a thorough examination of the eight limbs is not possible, but The Yoga Journal is a good resource for a lay interpretation of yoga's eight limbs. The practice of yoga is rooted in Hindu and Buddhist philosophies but is becoming more and more 
secularized in the United States, and most Americans associate yoga with asana practice (the physical postures), the third limb of yoga. Yet, asana is more than a physical posture. Traditionally, the asanas were developed to make the body limber and flexible enough to sit comfortably in meditation or other spiritual exercises for long periods. Hence, the first element of the union, the body, is the focus of asana practice.

The second element of the union is the mind. According to Patanjali's Yoga Sutras, chitta vritti nirodha is the goal of yoga [14]. Chitta vritti nirodha, another Sanskrit phrase, loosely interpreted, means to still or to calm the fluctuations of the mind. The eight limbs of yoga emphasize mental focus, concentration, meditation, mental clarity, and fundamentally cultivate what we know today as mindfulness. This may be demonstrated while a yoga practitioner is in a balancing asana, for example, and is instructed by the teacher to apply drshti (another Sanskrit term), or to focus his or her gaze on a particular point, which helps to improve concentration while holding a pose, especially a balancing posture. The mind and the body work together to cultivate a state of physical and mental balance while experiencing a physically challenging posture.

The final element, breath, may be the most crucial. In yoga class, one often hears the term pranayama or pranayam. Pranayam is the fourth limb of yoga and refers to the breath, or the life force-the vital energy, or spirit. One is often guided to practice pranayam in a yoga class. Though there are many forms of pranayama, it is typically demonstrated in a yoga class through deep and peaceful breathing, and/or allowing the breath to guide the movement in and out of the asanas. The result of this union between body, mind, and breath is a moving meditation.

The goal for many yogis is to reach the eighth limb of yoga, Samadhi - "Perfect union of the individualized soul with infinite spirit. A state of oneness; complete absorption" [15](para 1). Underlying this union is a philosophical system based on cultivating compassion, service, nonharming, non-judgment, detachment from the ego, and integrity, among other ethical or spiritual principles. The evidence base for yoga is growing, and it has been found to be helpful for numerous physical and behavioral health conditions. This report focuses specifically on addiction.

\section{Mindfulness and Addiction Recovery}

Because a substantial literature exists regarding mindfulness-based approaches to addiction treatment and recovery, and the yoga literature is still in its infancy, it may be best to begin here. Maybe the most well-known and researched intervention is Jon Kabat Zin's mindfulness-based stress reduction (MBSR), a model that includes yoga as one of the three primary techniques. MBSR has been shown to reduce stress, anxiety, and depression; enhance immune function; improve quality of sleep; lessen chronic pain-and the list goes on [16]. MBSR has been researched for well over 30 years now and is the parent model for several evidence-based, mindfulness-based interventions (MBIs) for treating addiction, such as mindfulness based cognitive therapy (MBCT), mindfulness-based relapse prevention (MBRP), and mindfulness-oriented recovery enhancement (MORE).

Yoga's main effect on addiction recovery may be through its impact on cognition, particularly craving and negative mood states. As noted previously, yoga fundamentally cultivates mindfulness. Both MORE and MBSR specifically affect addiction recovery through mindfulness. For example, the first core process of MORE is mindfulness. According to Eric Garland [17], developer of MORE, this process teaches individuals three things: (1) to notice when their attention focuses on psychological 
or environmental cues/stressors (triggers); (2) to learn how to shift the attention away from craving and other negative affective states toward sensory processing; and (3) anchoring attention to the breath through the process of mindful breathing. Garland describes MORE as an approach to addiction recovery that combines mindfulness with the principles of positive psychology and cognitive therapy.

The other intervention that deserves mention regarding its effect on craving is MBRP. Urge surfing is a MBRP technique that specifically targets craving. It teaches individuals to observe the sensation of craving, without judgement, and to simply ride it like a wave. As a wave ebbs and flows with the tide, so does craving. Several studies have shown MBRP to have a positive effect on craving [18-20].

\section{Yoga and Addiction Recovery}

The past decade has produced a number of systematic reviews examining yoga as a modality for addiction recovery. A 2014 review regarding the use of yoga in addiction treatment examined the outcomes of eight randomized controlled trials testing yoga's impact on a variety of substance use disorders [21]. Three of the RCTs examined yoga's effect on smoking cessation. The first showed statistically significant abstinence rates at 8 weeks in the yoga group compared with the control group. Two RCTs found a significant effect on reduced cravings for nicotine, and one of the studies also showed decreased negative affect in the yoga group. Three RCTs examined the effects of yoga on patients with alcohol use disorders (AUDs). All three found statistically significant benefits for the yoga groups. For example, one study reported that 80 percent of patients with an AUD improved or recovered compared with 48 percent of the control group. Another study showed significant improvements in several areas among alcohol-dependent patients who practiced yoga: Mood, stress reduction, quality of life, appetite, and alcohol use. The third RCT with an AUD sample found that yoga had a significant anti-depressant effect on the sample. Similarly, a study with 24 men with SUD showed statistically significant reductions in depression and anxiety among those who practiced yoga. Only one of the eight RCTs found no statistically significant difference between research and control groups. This group was in a methadone maintenance program, and the authors concluded that traditional methadone treatment programs were more effective than alternatives. The researchers found the results to be encouraging for yoga as an intervention or adjunct to addiction treatment and recovery.

A more recent review had similar findings [22]. These researchers included 16 studies, 12 of which were RCTs. Sample sizes ranged between 18 and 624. The authors also concluded that yoga appears to be an effective tool in addiction treatment and recovery. Yoga's positive effects on craving, stress, anxiety, depression, and negative mood may prevent a return to substance use for those who practice yoga and are recovering from addiction. However, both reviews found that most research has been conducted on nicotine use disorders and alcohol use disorders.

The most recent reviews $[23,24]$ came to similar conclusions, but included a wider range of substances used, though alcohol and nicotine are the most reported substances. Brooks and colleagues [23] examined 10 RCTs with participants reporting alcohol, nicotine, and other drug use. This review focused on yoga's effectiveness as part of a treatment program for women who have SUDs. The investigators reported outcomes equal to or favorable to the comparison groups for yoga as an adjunct to SUD treatment. In the latest review [24], the primary outcome variables were 
anxiety, pain, and craving. The substances involved were alcohol, tobacco, and opioids. Seven of eight studies reported significantly positive outcomes on outcome variables.

The conclusions and limitations reported by these researchers are congruent. Further research is needed to address yoga's efficacy with a wider range of substance use disorders. Heterogeneity in the research regarding the styles of yoga used (Bikram, Ashtanga, Vinyasa, lyengar, etc.), the number of yoga sessions study participants attended, small sample sizes, methodological flaws and deficits, make generalizability problematic.

Overall, yoga appears to be safe and effective as an adjunct to addiction treatment and recovery, and findings are encouraging for yoga as method of SUD treatment, but larger studies and more RCTs are needed for recommendation as a standalone intervention. More rigorous study is needed with larger sample sizes and more sophisticated research designs [22, 23, 25].

(See Yoga Alliance [25] for a comprehensive list of yoga research.)

\section{Conclusions}

12-step addiction recovery mutual aid groups are the most ubiquitous recovery groups in the world and their members often talk about the obsession to use (craving), and this might be where yoga's most powerful effect lies. The research on yoga and addiction recovery suggest that yoga may quiet cravings, and cravings may be the most powerful risk factor for a return to substance use [17-23].

Negative mood is another significant risk factor for relapse, and studies also support yoga's positive impact on mood states [22, 23]. Although the research regarding yoga as a modality for addiction treatment and relapse prevention is encouraging, and there are substantial limitations in the body of research, evidence to date suggests yoga is a safe, effective, holistic approach as an adjunct to addiction treatment and recovery.

\section{Author Contributions}

The author did all the research work of this study.

\section{Competing Interests}

The author has declared that no competing interests exist.

\section{References}

1. American society of addiction medicine. Definition of addiction [Internet]. United States: ASAM; 2019. Available from: https://www.asam.org/Quality-Science/definition-of-addiction.

2. Van Wormer K, Davis DR. Addiction treatment. Boston: Cengage Learning; 2016.

3. Narcotics A. Narcotics anonymous. 6th ed. Chatsworth: World Service Office; 2008.

4. American psychiatric association. Diagnostic and statistical manual of mental disorders. 5th ed. Washington: American Psychiatric Association; 2013.

5. Schneekloth TD, Biernacka JM, Hall-Flavin DK, Karpyak VM, Frye MA, Loukianova LL, et al. Alcohol craving as a predictor of relapse. Am J Addict. 2012; 21: s20-s26.

6. Alcoholics anonymous. 3rd ed. New York: Alcoholics Anonymous; 1976. 
7. Garland EL, Howard MO. Mindfulness-based treatment of addiction: Current state of the field and envisioning the next wave of research. Addict Sci Clin Pract. 2018; 13: 1-14.

8. Fitzgerald C, Barley R, Hunt J, Klasto SP, West R. A mixed-method investigation into therapeutic yoga as an adjunctive treatment for people recovering from substance use disorders. Int J Ment Health Addict. 2021; 19: 1330-1345.

9. Kuppili PP, Parmar A, Gupta A, Balhara YP. Role of yoga in management of substance-use disorders: A narrative review. J Neurosci Rural Pract. 2018; 9: 117-122.

10. Khanna S, Greeson JM. A narrative review of yoga and mindfulness as complementary therapies for addiction. Complement Ther Med. 2013; 21: 244-252.

11. Mallik D, Bowen S, Yang Y, Perkins R, Sandoz EK. Raja yoga meditation and medication-assisted treatment for relapse prevention: A pilot study. J Subst Abuse Treat. 2019; 96: 58-64.

12. Sarkar S, Varshney M. Yoga and substance use disorders: A narrative review. Asian J Psychiatr. 2017; 25: 191-196.

13. Yoga Journal, Yoga Alliance. 2016 yoga in America study conducted by yoga journal and yoga alliance [Internet]. Boulder: Sharon Houghton; 2016. Available from: https://www.yogajournal.com/yogainamericastudy/.

14. Satchidananda S. The yoga sutras of patanjali: Translation and commentary by Sri Swami Satchidananda. Buckingham: Integral Yoga Publications; 2014.

15. Ananda. The yoga encyclopedia [Internet]. California: Ananda Yoga; 2021. Available from: https://www.ananda.org/yogapedia/samadhi/.

16. Center for mindfulness. FAQ'S [Internet]. UMass Memorial Health Center for Mindfulness; 2017. Available from: https://www.ummhealth.org/umass-memorial-medical-center/servicestreatments/center-for-mindfulness/faqs.

17. Garland E. Mindfulness-oriented recovery enhancement for addiction, stress, and pain. Washington: NASW Press; 2013.

18. Li W, Howard MO, Garland EL, McGovern P, Lazar M. Mindfulness treatment for substance misuse: A systematic review and meta-analysis. J Subst Abuse Treat. 2017; 75: 62-96.

19. Vadivale AM, Sathiyaseelan A. Mindfulness-based relapse prevention-A meta-analysis. Cogent Psychol. 2019; 6: 1567090.

20. Grant, Sean DPhil; Colaiaco, Benjamin MA; Motala, Aneesa BA; Shanman, Roberta MS; Booth, Marika MS; Sorbero, Melony PhD; Hempel, Susanne PhD Mindfulness-based Relapse Prevention for Substance Use Disorders: A Systematic Review and Meta-analysis, Journal of Addiction Medicine: September/October 2017 - Volume 11 - Issue 5 - p 386-396. doi: 10.1097/ADM.0000000000000338

21. Posadzki P, Choi J, Lee MS, Ernst E. Yoga for addictions: A systematic review of randomised clinical trials. Focus Altern Complement Ther. 2014; 19: 1-8.

22. Kuppili PP, Parmar A, Gupta A, Balhara YP. Role of yoga in management of substance-Use disorders: A narrative review. J Neurosci Rural Pract. 2018; 9: 117-122.

23. Brooks J, Lawlor S, Turetzkin S, Goodnight C, Galantino ML. Yoga for substance use disorder in women: A systematic review. Arch Phys Med Rehabil. 2020; 101: e114-e115.

24. Walia N, Matas J, Turner A, Gonzalez S, Zoorob R. Yoga for substance use: a systematic review. J Am Board Fam Med. 2021; 34: 964-973.

25. Yoga Alliance. Other mental health conditions [Internet]. Arlington: Yoga Alliance; 2021. Available from: 
https://www.yogaalliance.org/About_Yoga/Scientific Research_on_Yoga/Disease_and_Disor ders/Other Mental Health Conditions.

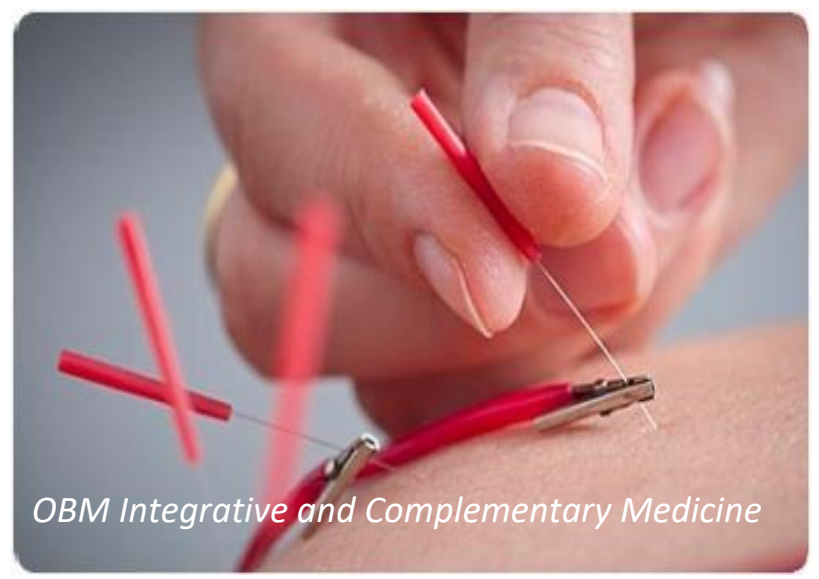

Enjoy $O B M$ Integrative and Complementary Medicine by:

1. Submitting a manuscript

2. Joining in volunteer reviewer bank

3. Joining Editorial Board

4. Guest editing a special issue

For more details, please visit:

http://www.lidsen.com/journals/icm 